\title{
Against Chalmers' Epiphenomenalism
}

\section{Glenn Braddock}

State University of New York at Albany

\section{Introduction}

There have been some recent attempts by philosophers to convince us to take seriously the threat of epiphenomenalism about consciousness. Ned Block [1995] argues that many of the common arguments in favor of phenomenological causation are fallacious because the conclusion that phenomenal consciousness ( $\mathrm{P}$ consciousness) is causal is reached based on evidence which only shows that direct access to information (A-consciousness) serves some function. David Chalmers [1996] develops an argument for epiphenomenalism based on similar considerations. For Chalmers, it is the "direct availability for global control" [Chalmers 1996, p. 225] of information that does all of the work, while the associated phenomenal "feel" plays no part in behavior. I will argue that there is no way to generate an argument for epiphenomenalism out of the conceptual distinction between access-consciousness and phenomenal consciousness without begging the question. In fact, the tight empirical link between access-consciousness and phenomenal consciousness, instead of leading us toward epiphenomenalism, should only strengthen our conviction that phenomenological properties are causal properties.

\section{The hard problem}

For Chalmers, as for Block, we can distinguish two senses of "consciousness". There is first of all phenomenal consciousness, for which Chalmers suggests we ought to reserve the name "consciousness". This is the puzzling "what it's like" character of experience. Then there is psychological consciousness, which Chalmers calls "awareness" and characterizes as the "direct availability for global control" [Chalmers 1996, p. 225]. "Aw'areness can be broadly analyzed as a state wherein we have access to some 
information, and can use that information in the control of behavior...This is clearly a functional notion" [ibid, p. 28]. While there is lots of scientific work to be done before we have a complete account of this kind of access, explaining awareness poses no metaphysical mysteries. Discovering the nature of attention, control of behavior, reportability, etc. are the "easy problems" of consciousness. "All of them are straightforwardly vulnerable to explanation in terms of computational or neural mechanisms" [Chalmers 1995, p. 10]. The "hard problem" arises when we try to explain consciousness as such. Why is awareness accompanied by phenomenal consciousness? "It is widely agreed that experience arises from a physical basis, but we have no good explanation of why and how it so arises. Why should physical processing give rise to a rich inner life at all?" [ibid, p. 11].

Like Block, Chalmers thinks that the failure to recognize this distinction between awareness and consciousness, and thus the difference between the easy and the hard problems, has resulted in premature optimism about the possibility of a solution to the mystery of consciousness. According to Chalmers, theorists often claim to be explaining full-blooded phenomenal consciousness when they only address different aspects of awareness: "At the close, the author declares that consciousness has turned out to be tractable after all, but the reader is left feeling like the victim of a bait-and-switch. The hard problem remains untouched" [ibid]. This sounds very similar to Block's complaint that a story about the function of Aconsciousness does not lead immediately to an account of the function of P-consciousness. But Chalmers uses this worry as the foundation for a full-blown argument for epiphenomenalism.

\section{From zombies to epiphenomenalism}

We should note that Chalmers is hesitant to embrace the term "epiphenomenalism", offering the following explanation: "I do not describe my view as epiphenomenalism. The question of the causal relevance of experience remains open, and a more detailed theory of both causation and of experience will be required before the issue can be settled" [Chalmers 1996, p. 160]. But his reasons for this qualification do not question the spirit of the epiphenomenalist 
argument at all. For example, he flirts with the idea of panpsychism: "Perhaps, as Russell suggested, at least some of the intrinsic properties of the physical are themselves a variety of phenomenal property?" [ibid, p. 154]. If this were the case, then phenomenal properties would be causally relevant since they would be central to the physical constitution of the world. Still, my conscious experiences as subjective states of my mind would not make any difference in the world. Chalmers admits that "the view makes experience explanatorily irrelevant. We can give explanations of behavior in purely physical or computational terms, terms that neither involve nor imply phenomenology" [ibid, p. 156]. Chalmers says this only "looks something like epiphenomenalism" [ibid, p. 150], or "feels epiphenomenalistic" [ibid, p. 156], describing his view as "epiphenomenalistic to a first approximation" [ibid]. I think a view according to which consciousness makes no difference in behavior can fairly be described as epiphenomenalistic through and through, but whatever it should be called, it is to Chalmers' case for this position that I now turn.

Armed with what I consider the reasonable conviction that consciousness cannot be functionally or physically defined, Chalmers puts forth the following argument against the logical supervenience of consciousness:

Whatever functional account of human cognition we give, there is a further question: Why is this kind of functioning accompanied by consciousness? No such further question arises for psychological states. If one asked about a given functional model of learning, "Why is this functioning accompanied by learning?" the appropriate answer is a semantic answer: "Because all it means to learn is to function like this". There is no corresponding analysis of the concept of consciousness. Phenomenal states, unlike psychological states, are not defined by the causal roles that they play. It follows that explaining how some causal role is played is not sufficient to explain consciousness. After we have explained the performance of a 
given function, the fact that consciousness accompanies the performance of the function (if indeed it does) remains quite unexplained.

... no matter what functional account of cognition one gives, it seems logically possible that that account could be instantiated without any accompanying consciousness. [ibid, pp. 46-47]

This is to accept the logical possibility of a philosophical zombie:

What is going on in my zombie twin? He is physically identical to me, and we may also suppose that he is embedded in an identical environment. He will certainly be identical to me functionally: he will be processing the same sort of information, reacting in similar ways to inputs, with his internal configurations being modified appropriately and with indistinguishable behavior resulting... It is just that none of this functioning will be accompanied by any real conscious experience. There will be no phenomenal feel. There is nothing it is like to be a zombie. [ibid, p. 95]

And this, he thinks, leads straight to epiphenomenalism:

The basic shape of the argument is clear: if it is possible to subtract the phenomenal from our world and still retain a causally closed world Z, then everything that happens in $Z$ has a causal explanation that is independent of the phenomenal, as there is nothing phenomenal in Z. But everything that happens in $\mathrm{Z}$ also happens in our world, so the causal explanation that applies in $\mathrm{Z}$ applies equally here. So the phenomenal is causally irrelevant. Even if conscious experience were absent, the behavior might have been caused in exactly the same. [ibid, p. 150] 
This case for epiphenomenalism can be summarized as follows: Absent qualia zombies are possible. This means that there could be a creature which lacks consciousness but which has the same functional structure as I have. It is therefore possible that the same causal roles could be performed without any phenomenology. It is possible, then, for phenomenological properties to be epiphenomenal. This means, though, that there is a possible world in which everything happens exactly as it happens in the actual world, but in which everything can be explained without reference to consciousness. But since the two worlds are functionally identical, then the same functional account explains the happenings of both worlds. Therefore, everything that occurs in the actual world can be explained without appeal to consciousness. This means that consciousness plays no causal role in the actual world.

The weakness of this argument is with the first premise which states that absent qualia zombies are possible. As Block has pointed out, this claim is ambiguous. It could be given the following three readings:

Absent Qualia Premise, Strong Reading: If absent qualia are possible, then pain could lack qualitative character, and its lacking qualitative character would make no difference to its causal role. [Block 1980, p. 265]

AQ Premise, Weak Reading: If absent qualia are possible, there could be ersatz pain that has the same causal role as pain. [ibid, p. 266]

AQ Premise, Weaker Still Reading: If absent qualia are possible, ersatz pain is possible. [ibid, p. 271]

According to the strong reading, $m y$ pains could lack their qualitative character without any change in my mental function; according to the weak reading, some creature's ersatz pain could lack qualitative character and yet have the same (total) causal powers as my hurtful pain; according to the still weaker reading, this other creature's ersatz pain could play the same role in its mental life as 
my hurtful pain plays in mine, yet the total causal powers of our pains might differ (e.g., my pain and its ersatz pain might result in the same behavior in like circumstances but have different effects on an EEG [ibid]).

These distinctions are crucial here because Chalmers needs the strong reading in order for the argument for epiphenomenalism to go through. The two weaker AQ premises provide no support whatsoever for epiphenomenalism. If either of these weaker readings is true, it only shows that something else could have exactly the same effects (limited to the context of psychological explanation on the weak reading; in all contexts on the still weaker reading) as my phenomenological states. But the fact that something else could do the job does not make the thing that actually does the job an epiphenomenon. To take one of Block's illustrations, "mousetraps with springs can be functionally identical to mousetraps without springs. (So an "absent spring" hypothesis is true.) But it would be silly to conclude that the presence or absence of springs make no difference to the causal consequences of the states of those mousetraps that have springs" [ibid, p. 262]. Furthermore, even if it were true that the same functional explanation could account for both my behavior and the behavior of my zombie twin, this would not necessarily entail that my conscious states are epiphenomenal. It might be the case that I would behave exactly the same if the phenomenal could be subtracted from my mental life, and yet the phenomenal could still be causal. The presence of the qualitative character might, for example, affect the manner in which the behavior is caused. In such a case, my phenomenal states would be causal, even though the functional explanation of my zombie twin could equally account for my behavior.

Chalmers seems to recognize this when he describes his zombie twin as physically, functionally, and contextually identical to himself, lacking only consciousness. If this were possible, then the threat of epiphenomenalism would be more serious. If for example, my zombie twin's body and environment are physically identical to my own, then it cannot be that my phenomenal states are causing the same behavior in a different way, because that difference would have to turn up in some manner in the physical processes of our bodies or of our worlds. But this is ruled out by how 
Chalmers sets up the thought-experiment. Epiphenomenalism about consciousness becomes a real worry if the absence of consciousness would not change behavior or the way that the behavior is caused.

The problem for Chalmers is that, far from showing that the strong version of the AQ premise is true, he never gets beyond the weakest version of the premise, the idea that there could be ersatz pain. The only support he can give for the strong AQ premise, besides his insistence that it just seems obvious to him, is the "indirect support" from "considering nonstandard realizations of my functional organization" [Chalmers 1996, p. 97]. That is, he tries to use the plausibility of the idea that ersatz pain is possible to pump our intuitions toward the idea that $m y$ pain could function the same without its qualitative character. So Chalmers mentions Block's idea that "the people of a large nation such as China might organize themselves so that they realize a causal organization isomorphic to that of my brain" [ibid]; we might also consider "my silicon isomorph, who is organized like me but who has silicon chips where I have neurons" [ibid]. These examples, while farfetched, indeed seem like open possibilities. But now consider the moral that Chalmers draws from these possibilities:
All that matters here is that the idea that such a system lacks conscious experience is coherent. A meaningful possibility is being expressed, and it is an open question whether consciousness arises or not... Whether such an isomorph would in fact be conscious is controversial, but it seems to most people that those who deny this are expressing a coherent possibility. From these cases it follows that the existence of my conscious experience is not logically entailed by the facts about my func- tional organization [ibid].

If this last sentence is interpreted to mean that the existence of conscious experience just like mine is not logically entailed by the facts about my functional organization, then it would seem that this conclusion is supported by the possibility of nonstandard real- 
izations of my functional organization. If a silicon, nonconscious functional duplicate of me is possible, then clearly consciousness does not logically supervene on functional structure. But why is it plausible that my silicon functional isomorph might lack consciousness? Because if we accept the supervenience of the mental on the physical, the idea that there is no mental difference without a physical difference, then we hold the different lower-level implementations of our minds responsible for the mental difference between me and my zombie twin. But then Chalmers claims that these first thought-experiments, if we think that they represent possible scenarios, ought to make us think that I could have a zombie functional twin which is also physically identical to me:

For it is clear that there is no more of a conceptual entailment from biochemistry to consciousness than there is from silicon or from a group of homunculi. If the silicon isomorph without conscious experience is conceivable, we need only substitute neurons for silicon in the conception while leaving functional organization constant, and we have my zombie twin. Nothing in this substitution could force experience into the conception; these implementational differences are simply not the sort of thing that could be conceptually relevant to experience. So consciousness fails to logically supervene on the physical. [ibid]

This drastically changes what we are supposed to imagine. It was the physical differences that made the original cases make sense. Now Chalmers asks us to do away with any physical difference, whether local or global, between me and my zombie twin, and then he suggests that this is just as coherent a possibility as the original case. But if we accept this second possibility, we have already accepted that consciousness is epiphenomenal from the start. At this point, though, Chalmers is supposed to be giving us a reason to think that the first premise in the argument for epiphenomenalism, the strong $A Q$ premise, is true. 
Chalmers maintains that the logical possibility of his zombie twin as he has described it, a being both physically and functionally identical to him but lacking consciousness, seems perfectly obvious and "the burden is on the opponent to give us some idea of where the contradiction might lie in the apparently quite coherent description" [ibid, p. 99]. But what we have seen is that the AQ premise is only compelling when taken in one of its weaker senses, and the acceptance of either of these weaker premises does not commit you to the strong premise. If, say, the weakest premise is true so that ersatz pain is possible, which seems at least prima facie plausible to me, then Chalmers is right when he makes the general claim that it is logically possible for a functional isomorph of me to lack consciousness. But it still may be logically impossible for a physical, functional, and contextual duplicate of me to lack consciousness, because $m y$ pains might not be able to perform their functions without their qualitative character. Even if a silicon, nonconscious, functional duplicate of me is possible, this grounds no conclusions about the potency of my phenomenological states. My zombie twin's silicon constitution might make all the difference in the world. Maybe more information can be stored and efficiently processed in silicon chips than in neurons, so that he can get by without consciousness, while I need consciousness to function as I do with the neural equipment that I have.

We are in a position, then, to give a simple response to Chalmers' challenge. How might it be logically impossible for a physical and functional duplicate of me to lack consciousness? Just in case the correct functional characterization of me requires consciousness, that is, just in case epiphenomenalism is false about $m y$ phenomenological states. Chalmers claims again and again that "no matter what functional account of cognition one gives, it seems logically possible that that account could be instantiated without any accompanying consciousness" [ibid, p. 47, my italics]. But this is just false if consciousness plays a functional role. If this is the case, then it would be logically impossible for the functional account to be instantiated without consciousness. Of course, it would not be logically impossible in the same way that it would be logically impossible for a functional account of learning to be instantiated without any learning. The learning just is the 
function, while the consciousness would not be exhausted by the function. Still, if the functional account includes a phenomenological state in its explanation of some behavior, then it would be logically impossible for that behavior to occur without the phenomenological state. Why, at this point, can I imagine that any function could be accomplished without consciousness? Simply because our functional accounts are so incomplete. It may turn out that we can give a complete functional account of the mind without including consciousness, but it may not. We have to wait to see what the functional account requires before ruling out $a$ priori that consciousness matters.

The upshot is that Chalmers' argument for epiphenomenalism begs the question. He argues that, since it is imaginable and thus logically possible for there to be a physical and functional duplicate of me that lacks consciousness, and since all of my zombie twin's behavior can be explained without reference to consciousness, then all of my behavior, which is identical to my twin's, can be explained without reference to consciousness. But how can this justify epiphenomenalism when the first premise openly assumes it? To say that any functional account of my behavior might be implemented in a nonconscious being is just to say that none of my phenomenological states make a difference. It may turn out that the functional account of any particular mental process will not require reference to consciousness. This, though, is only imaginable because, as Block puts it, the concept of phenomenal consciousness is not a functional concept.

The failure of Chalmers' position is evident from the beginning, since the way he carves up the problems of consciousness is itself question-begging. He assumes throughout that the "easy problems" can fall without a theory of phenomenal consciousness, which is just to assume that consciousness plays absolutely no causal role. He asks, "How do we explain the performance of a function? By specifying a mechanism that performs the function. Here, neurophysiology and cognitive modelling are perfect for the task" [Chalmers 1995, p. 12]. But the question is whether or not it is possible to fully explain the mechanism without appealing to subjective consciousness. If not, then, as David Hodgson puts it, “the easy problems ain't so easy" [Hodgson 1996]. 
Chalmers slides illicitly from the fact that the completed functional account of the mind, the one that we in fact settle on, might not need to appeal to any conscious states to the claim that any functional account whatsoever could get by without consciousness. This rules out phenomenological causation right from the gate, a move that will not convert anyone to epiphenomenalism.

\section{Phenomenal access}

We can do more, though, than attack arguments for epiphenomenalism that draw unjustified conclusions from the perfectly good distinction between access and experience. We can, for instance, draw out the implications of the tight empirical link between these different aspects of consciousness. Without resorting to imaginary cases, it seems impossible to find any cases where direct availability of information for global control, the kind necessary for reportability and rational use of the information, is present without subjective consciousness. Likewise for cases of experience without access. This certainly requires an explanation. In response to one of Block's suggested explanations of this empirical bond, Chalmers writes: "Block suggests that P-consciousness might "grease the wheels" of A-consciousness, but this cannot work. P-consciousness is redundant to the explanation of the physical mechanisms of A-consciousness, as the conceivability of the zombie shows: same physical mechanisms, same explanation of A-consciousness, no P-consciousness" [Chalmers 1997, p. 423]. But we have now seen that Chalmers has no right to make this conjecture. The zombie thought-experiment already depends on the redundancy of P-consciousness, so it cannot be used to establish the redundancy of P-consciousness. Thus we are free to explore the possibility that the striking correlation between access and experience is not a mere correlation, but that P-consciousness, in creatures like us anyway, is necessary for the kind of direct availability for global control that epiphenomenalists claim could go on in the dark.

Robert Van Gulick argues that understanding the role of consciousness requires more than understanding the inputs and the 
end results of mental processes that involve conscious states. We also need to know how consciousness does its work:

Nonconscious states might be able to make a similar contribution in the context of at least some systems... But insofar as such nonconscious states differ from our conscious states in the processes by which they make their equivalent contribution, there is an important respect in which they differ in their functional roles. [Van Gulick 1994, p. 27]

As any cursory examination of the progress of artificial intelligence would indicate, there is no machine that even comes close to the capacities of humans, and other animals, for efficient access to the information necessary to successfully navigate our way through the world. One apparent difference between us and machines is that we are conscious. Without committing ourselves to the premature conviction that machines could not be given these abilities without making machines conscious, it is worth considering the possibility that consciousness is the thing, or more plausibly just one of the things, that happens to give us the edge.

But what is so special about (phenomenal) consciousness that it should be such an advantageous feature of our mental lives? Van Gulick suggests that the distinctive character of phenomenal representations can be understood in terms of their semantic transparency. "Indeed they are so transparent that we typically "look" right through them. Our experience is the experience of a world of familiar objects-of desks, chairs, coffee cups, and beech trees. Moreover this transparency is to some extent an immediately experienced feature of our conscious life..." [Van Gulick 1997, p. 438]. He explains further:

Any phenomenal object is delimited and defined within the representation in large part through the relations that it bears to other objects, which are in turn defined in part by the relations that they bear to it. It is in part the density of these interdefining relations that gives phenomenal ob- 
jects their "thickness", their objectivity. The fact that the phenomenal representation of objects involves such dense and interdependent relations might help to account for its high degree of semantic transparency; any phenomenal representation of an object would of necessity also be a representation of its myriad relations within its world. [ibid, pp. 438-439]

The general idea seems to be that conscious representation of the world best reflects the complex structure of that world, so much so that consciousness thrusts us right into the world. To borrow a phrase from Hubert Dreyfus, consciousness allows us to be "in a situation" in virtue of the density of its deliverances from the environment. To successfully navigate through the world and respond appropriately to its changes we need to employ mental representations with sufficiently rich content to quickly and efficiently relay detailed information about large chunks of the world. Phenomenally conscious mental representations seem to be perfect for the job:

Sensuous manifolds provide a medium well suited for the representation of such rich and easily accessed spatio-temporal information. They have a continuous structure isomorphic to the spatio-temporal domains they are used to represent. Thus by using qualia to delimit regions of such manifolds as representing objects, it is possible to implicitly represent a large stock of information about the relative spatio-temporal relations of those objects. [ibid, p. 439]

Consider the difference between trying to reach a completely unfamiliar location by using a list of directions and trying to get there by using a road map. If, unexpectedly, you should find a road closed due to construction, your directions may become useless, but with your map you will be able to quickly and easily identify an alternative route. This is because the map provides a thicker 
representation of the environment, conveying more detailed information in a very efficient manner. When it comes to making our way through the world, a picture is truly worth a thousand words. The "pictures" offered by conscious experience are so rich and detailed that they seem nothing like representations at all. From a phenomenological standpoint, the world is immediately present to mind. This proves extraordinarily helpful when it comes to complex activities like driving a car, blocking a speeding hockey puck, or hunting down dinner.

Of course, appealing to the semantic transparency of conscious representations cannot close the matter about the function of consciousness. As Van Gulick admits, there is no reason to conclude a priori that representations with sufficiently rich content to allow human-like behavior could not be implemented in a machine which lacks phenomenal consciousness. But, in our own case, the firstperson perspective reveals a fertile stream of consciousness that is, as Van Gulick puts it, a world in its own right. This makes phenomenal consciousness a great candidate for the kind of representational system required for direct availability for global control.

It could be that phenomenally conscious states simply mirror nonconscious states that display the same semantic transparency. Ultimately, this skeptical conjecture will probably stand or fall with empirical evidence. For example, will we find that there is something about consciousness that, for creatures like us, makes it a particularly useful vehicle for information about the world, some feature that nonconscious states could not provide for us, or could only provide at too great a cost? Block speculates that the reason phenomenal consciousness was selected for by evolution is that it plays some special role in motivation [Block 1991, p. 670]. A very effective way to convey information about tissue damage to an organism is to make that damage hurt, and a very effective way to minimize tissue damage is to make the organism aversive to hurtfulness. It's not the only way. Mother nature might have designed us so that we detected bodily damage through external perception, like a sailor detects damage to his ship, to use Descartes' image. It seems plausible, though, that there is something about the kind of creatures we are-perhaps it is the kind of stuff we are 
made of, or how small our heads must be so they will not be too cumbersome, or the nature of the environment in which we are embedded-that makes it especially advantageous for the world to feel and seem certain ways to us. We'll have to wait and see, but the tight empirical bond between access and experience, and the fact that consciousness seems so well-suited to provide a rich model of a complex world, should lead us to suspect that the epiphenomenalist's skeptical suspicions will remain ungrounded.

\section{Phenomenological potency and irreducibility}

I am not sure that the metaphor of experience "greasing the wheels" of access-consciousness is the most appropriate. It is better to think of phenomenal consciousness as a particularly greasy wheel in its own right. This in no way threatens the idea that phenomenological properties are irreducible. As Chalmers recognizes, "Although conscious states may play various causal roles, they are not defined by their causal roles. Rather, what makes them conscious is that they have a certain phenomenal feel, and this feel is not something that can be functionally defined away" [Chalmers 1996, p. 105]. It could be, then, that every particular phenomenological mental event is a functional event, with potency in virtue of its phenomenological properties, although the functional account of that event, given in purely objective terms, will not capture those phenomenological properties.

Ron McClamrock suggests the following explanation which draws on "Husserl's distinction between noeses - the real temporal parts (or 'proper components') of an experience-and noemaan act's essential intentional character (or 'correlate')":

Even if the phenomenological globally supervenes on the computational, phenomenological properties needn't be coextensive with local syntactic properties of representations. Phenomenological properties could be context-dependent properties of those representations, or could be properties of groups, streams, or processes defined over representations that might be multiply realizable at the 
level of real token syntactic objects - that is, they might well be an irreducible higher level of organization of the system.

Taking a hint from seeing the noema as intentional correlate rather than proper part of the intentional act, we might similarly in the naturalistic case find the best account of the object-directed phenomenological state not in the unity of some particular representational state, but in the constancy of the object of experience. The intentional or phenomenological unities in experience-the taxonomy under which human action has its noticeable systematicities as rational and goal-directedneedn't be real, temporal parts of the flow of consciousness, but might be the (abstract, intentional) noematic unities of the real noetic phases of consciousness. The shift in grain between the noetic and the noematic suggests the possibility of a parallel shift between the computational and the phenomenological that leaves the latter as fundamentally non-reducible and autonomous [McClamrock (1995) pp. 181-183].

The suggestion here is that, when explaining some behavior, it may often turn out that the best explanation is one that is framed in terms of how the world seems to the subject, and how the world seems to the subject may not always be definable in terms of the physical or computational properties of the subject. In general, we can make a case for higher-level causation whenever the causation is in virtue of higher-level properties [ibid, p. 43]. We can make sense of phenomenological causation in the same way. Even though each phenomenological event is identical with some physical and computational events, it may often be that what matters for explaining some behavior is the character of the state considered as a subjective experience.

Following McClamrock's suggestion, consider the possibility that phenomenological properties are multiply realizable with re- 
spect to computational properties. A nice example of this is provided by Dennett (although Dennett himself certainly does not intend it to help demonstrate the irreducibility and causal potency of phenomenological properties, since he denies the existence of phenomenological properties). Dennett distinguishes between the content of a representation and the vehicle or medium of a representation. So we may see a particular region as having a certain arrangement of colors; this is the content of our perceptual experience. But the bearing of this content can be achieved in many ways. For example, the perceptual experience having that content might be accomplished by a mechanism something like color-bynumbers, where each region is labeled with a code for colors; alternatively, the mechanism involved might be color-by-bit-map, where the color is filled in pixel by pixel. Or it might be the case that color is represented by color, like in a photograph [Dennett 1991, p. 347-349].

If these are in fact legitimate possibilities, then we have a good example of how a particular kind of perceptual experience might be realized by many different computational states or processes. Now we can make sense of phenomenological causation in the same way we can make sense of any instance of higher-level causation, by asking whether there are any effects produced by this particular mental state that occur in virtue of its phenomenological properties. "Fix the properties more directly responsible for the effect, and then note the independence of the effect from the various ways in which those properties might be produced... by different lower-level implementations" [McClamrock 1995, p. 48]. So, taking another example from Dennett, imagine that you walk into a room and perceive the wallpaper as covered by hundreds of identical pictures of Marilyn Monroe [Dennett 1991, pp. 354-356]. As Dennett points out, this experience could be carried out in a number of ways, but must we say that whatever effects are brought about by the experience are effects in virtue of the details of the computational process that underlies it? It seems, on the contrary, that the multiple realizability of the experience shows that many effects of the state or process occur in virtue of its phenomenological character. For example, upon seeing the wallpaper, I might say, "Hey, nice wallpaper". If I had not perceived the wallpaper as 
covered by hundreds of identical Marilyns, if my perceptual experience did not have that particular phenomenological character, then I would not have uttered that sentence. But let's say that, in real life, the perceptual experience was accomplished by color-bynumbers. Now fixing the phenomenological character but changing the computational process to color-by-bit-map, would I have still said "Hey, nice wallpaper". Yes, because what was relevant about my mental state as I entered the room, in terms of its linguistic effect, was that it had the phenomenological character that it had, not that this phenomenological character was subserved by any particular computational process.

On this view, there is certainly a sense in which phenomenological properties are dependent on computational properties. If you could take a snapshot of a person's perceptual experience of a particular object or event in the world, one could, in principle, identify the real representational features of that experience at that time which contribute to the conscious experience overall; but phenomenological properties are causal in virtue of their unification of particular representations into the "perceived object as such", or in general, the perceived world as such. Objects can be perceived as the same objects throughout variations in the details of the particular representations that make up the perceptual experiences. As Van Gulick puts it, we most immediately perceive a "thick", unified world, not a world of discrete properties and events. McClamrock notes that it is in virtue of the stability of this world as perceived that much of our systematic behavior is to be explained; our behavior can, of course, only be explained by understanding how we perceive the world. A phenomenological account of consciousness would, ideally, specify the way the world seems to us as perceivers and agents who have to get around in that world [ibid, pp. 183-184]. That is, it would explain the unique nature of our phenomenological access to the world, an explanation that would certainly feed back into any functional account of our behavior. Phenomenological properties, then, depend, at least in part, on objectively describable functional properties, and they have effects on behavior in virtue of the way they present a unified world to the subject; and yet their multiple realizability by various particular representations and their context- dependence on the 
world in which the subject is embedded rule out their reducibility to objective physical or functional properties.

\section{References}

Block, N. (1980) “Are Absent Qualia Impossible?" Philosophical Review, 89, pp. 257-274.

Block, N. (1991) "Evidence Against Epiphenomenalism", Behavioral and Brain Sciences, 14, pp. 670-672.

Block, N. (1995) "On a Confusion About a Function of Consciousness", Behavioral and Brain Sciences, 18, pp. 227-247. Reproduced in Block, Flanagan, and Guzeldere (1997), pp. 375-415.

Block, N., Flanagan, O., and Guzeldere, G., eds. (1997) The Nature of Consciousness: Philosophical Debates. Cambridge: MIT Press.

Chalmers, D. (1995) "Facing Up to the Problem of Consciousness", Journal of Consciousness Studies, 2, pp. 200-19. Reproduced in Shear, J. (1997), pp. 9-30.

Chalmers, D. (1996) The Conscious Mind: In Search of a Fundamental Theory. Oxford: Oxford University Press.

Chalmers, D. (1997) "Availability: The Cognitive Basis of Experience?, in Block, Flanagan, and Guzeldere (1997), pp. 421-424.

Dennett, D. (1991) Consciousness Explained. Boston: Little, Brown, and Co.

Graham, G. and Stephens, G. Lynn, eds. (1994) Philosophical Psychopathology. Cambridge: MIT Press.

Hodgson, D. (1996) "The Easy Problems Ain't So Easy", Journal of Consciousness Studies, 3, pp. 69-75. Reproduced in Shear (1997), pp. 125-131.

McClamrock, R. (1995) Existential Cognition: Computational Minds in the World. Chicago: University of Chicago Press.

Shear, J., ed. (1997) Explaining Consciousness: The 'Hard Problem'. Cambridge: MIT Press.

Van Gulick, R. (1994) "Deficit Studies and the Function of Phenomenal Consciousness", in Graham and Stephens (1994), pp. 25-49.

Van Gulick, R. (1997) "Understanding the Phenomenal Mind: Are We All Just Armadillos? Part II: The Absent Qualia Problem", in Block, Flanagan, and Guzeldere (1997), pp. 436-442. 